\title{
Programmes et interventions favorisant l'équité en santé dans les populations LGBTQ2+ au Canada par des mesures sur les déterminants sociaux de la santé
}

\author{
Robert Higgins, B.A. (1,2); Brian Hansen, M.A. (1,2); Beth E. Jackson, Ph. D. (3); Ashley Shaw, M. Sc. (3); \\ Nathan J. Lachowsky, Ph. D. (1,2)
}

Diffuser cet article sur Twitter

\section{Résumé}

Les minorités sexuelles et de genre vivent un certain nombre d'inégalités en matière de santé. On sait que les déterminants sociaux de la santé sont à l'origine de ces iniquités, mais on dispose de peu de données probantes sur le nombre et les types d'interventions au Canada qui portent sur ces déterminants pour ces populations. Nous avons effectué une analyse des programmes disponibles au Canada qui ciblent les minorités sexuelles et de genre et nous les avons classés en fonction de leur niveau d'intervention (individuel/ interpersonnel, institutionnel et structurel). Nous avons constaté que peu de programmes ciblent les femmes, les adultes en milieu de vie, les Autochtones ou les minorités ethnoraciales, les immigrants et les réfugiés récents ou encore les locuteurs de langues minoritaires, et que peu d'interventions se déroulent à un niveau structurel.

Mots clés : minorités sexuelles et de genre, équité en santé, déterminants sociaux de la santé, santé des minorités, disparités d'état de santé, promotion de la santé

\section{Introduction}

Les personnes LGBTQ2 + * ont souvent une moins bonne santé physique et mentale que les personnes hétérosexuelles et cisgenres $^{1,2}$. Les disparités qu'elles connaissent sur le plan de la santé physique vont d'un mauvais état de santé général à des taux supérieurs de cancer, de maladies cardiovasculaires, d'asthme, de diabète, d'arthrite et d'autres maladies chroniques ${ }^{1}$. Les jeunes transgenres présentent également des disparités en matière de santé mentale, notamment un risque plus élevé de signaler une détresse psychologique, des automutilations, des épisodes dépressifs graves et le suicide ${ }^{2}$, qui sont des facteurs que l'on a pu corréler à des expériences de discrimination, de harcèlement et de violence ${ }^{3,4}$. Les jeunes LGBTQ2 + canadiens sont souvent victimes d'exclusion, d'isolement et d'anxiété ${ }^{\text {. On sup- }}$ pose que bon nombre des iniquités en matière de santé observées chez les minorités sexuelles et de genre (MSG) proviennent de la stigmatisation sociétale ${ }^{6}$, en particulier de la co-occurrence de stéréotypes, de l'étiquetage, de la perte de statut, de la séparation et de la discrimination $^{7,8,9}$ et d'expériences sociales négatives, qui créent un stress exacerbé ${ }^{1,2}$.

Les processus de stigmatisation et de discrimination jouent un rôle central dans les inégalités en matière de santé pour les populations de MSG, contribuant aux expériences de stress et de traumatisme tout au long de leur vie. Ils entraînent
Points saillants

- Il existe un certain nombre de lacunes dans les programmes favorisant l'équité en santé et les interventions visant les déterminants sociaux de la santé pour les minorités sexuelles et de genre au Canada.

- Les efforts visant à élaborer de nouveaux programmes devraient prendre en compte les communautés LGBTQ2 +, qui sont mal desservies par les services existants (en particulier les Autochtones, les minorités ethnoraciales, les femmes, les immigrants récents ou réfugiés).

- Très peu de programmes traitent de l'emploi, de l'invalidité, de l'éducation ou du logement, qui sont d'importants déterminants en amont de la santé.

- La plupart des programmes sont axés sur les niveaux d'intervention individuels et interpersonnels.

- Les interventions systémiques étant rares, les efforts devraient être axés sur l'étude des interventions structurelles déjà en place enfin d'en envisager l'extension.

* LGBTQ2+ est un acronyme générique utilisé dans cet article pour rendre compte de la diversité des orientations sexuelles et des identités de genre, notamment pour les personnes qui s'identifient comme lesbiennes, gaies, bisexuelles, transgenres (trans), queer ou qui sont bispirituelles autochtones. 
également un accès inéquitable aux ressources sociales et matérielles favorisant une bonne santé (emploi, revenu, logement, éducation suffisante et de qualité et soins de santé) ${ }^{10}$. Par exemple, $40 \%$ des 2873 répondants transgenres et non binaires à une enquête canadienne de 2019 vivaient dans un ménage à faible revenu et $45 \%$ ont déclaré avoir un ou plusieurs besoins en soins de santé non satisfaits au cours de l'année précédente ${ }^{11}$. Les femmes et les hommes bisexuels au Canada ont fait état de taux d'insécurité alimentaire de respectivement 2,8 et 2,5 fois plus élevés que leurs homologues hétérosexuels et de résultats de santé inférieurs à ceux de leurs pairs gais et lesbiennes ${ }^{12}$. Ces inégalités sont susceptibles d'être amplifiées pour les personnes dont l'orientation sexuelle ou l'identité de genre s'ajoute à d'autres identités sociales marginalisées, comme leur appartenance ethnique ou leur classe sociale ${ }^{13}$.

À ce jour, la plupart des recherches dans ce domaine ont porté sur les iniquités en santé, beaucoup moins de travaux portant sur le développement et l'évaluation des interventions ${ }^{6}$. Il n'existe donc pas de portrait complet des interventions visant ces déterminants chez les personnes LGBTQ2 + au Canada. C'est pour combler cette lacune que nous avons effectué une analyse de la conjoncture entre février et mars 2019. Nous avons mis à jour, en y ajoutant des entrées, la publication du rapport du Comité permanent de la santé du Parlement, La santé des communautés LGBTQIA2 au Canada, de juin 2019 ${ }^{14}$. Début 2020, nous avons communiqué nos résultats à certains organismes communautaires pour avoir un retour de la part de leurs membres.

\section{Méthodologie}

Une recherche systématique a permis de sélectionner des programmes axés sur les déterminants de la santé aux niveaux macro (structurel ou social, économique et politique), méso (institutionnel) et micro (individuel et interpersonnel). Nous avons également inclus certains programmes ciblant des comportements ou des résultats de santé précis. La recherche a été effectuée par province et territoire afin de rassembler les programmes à l'échelle du pays qui s'attaquent à un ou plusieurs des déterminants sociaux de la santé tout en ciblant les MSG. La recherche a exclu de fait les programmes visant des personnes ne relevant pas des minorités sexuelles ou de genre.

L’analyse préliminaire a révélé une abondance de programmes axés sur les considérations " en aval » et au niveau individuel (réduction des connaissances, attitudes et comportements individuels qui mènent à la stigmatisation ou à la discrimination et augmentation de la connectivité sociale). Compte tenu des associations importantes entre les inégalités en matière de santé et les conditions structurelles auxquelles font face les MSG, nous avons axé l'analyse sur les interventions en amont et intermédiaires. Nous avons donc exclu les programmes récréatifs en aval, comme les équipes sportives, les chorales et les cafés LGBTQ2 + , les alliances gaieshétérosexuelles en milieu scolaire, les programmes sociaux et les clubs offerts par les établissements postsecondaires, les églises et les institutions religieuses positivistes, les festivals de la fierté gaie et les événements ponctuels. (Les initiatives exclues par ces seuls critères justifieraient une analyse complète.) Cette analyse englobe donc les interventions à des niveaux plus élevés de l'écosystème social, que ce soit les interventions systémiques ou les interventions ciblant les déterminants sociaux de la santé (autres que la connectivité sociale) comme le manque d'accès à l'emploi, la stigmatisation et la discrimination, la pauvreté et l'insécurité alimentaire.

Premièrement, nous avons utilisé le moteur de recherche Google pour effectuer de vastes recherches sur Internet de sites en français et en anglais. Deuxièmement, nous avons effectué des recherches ciblées dans la base de données de l'Agence canadienne des médicaments et des technologies de la santé (ACMTS), les répertoires provinciaux 211 (fournissant de l'information sur les services communautaires et sociaux ainsi que des renvois à ces services) et les résultats ${ }^{\dagger}$ du financement des trois Conseils. Troisièmement, dans le but d'identifier des organismes, programmes et services communautaires, nous avons étudié les guides du festival de la fierté LGBTQ2 + de 2018 ainsi que les trois plus récents programmes de l'Association professionnelle canadienne pour la santé transgenre, du Sommet du Centre de recherche communautaire et des conférences de Santé arc-en-ciel Ontario. Enfin, nous avons effectué une analyse des bases de données universitaires à l'aide de Summon 2.0 (Université de Victoria, Victoria, C.-B.). Nous avons considéré nos recherches comme terminées lorsque deux pages subséquentes du site n'ont livré aucune information nouvelle ou pertinente.

L'information sur chaque programme a été traitée à l'aide du logiciel NVivo 11 (QSR International Pty Ltd., Melbourne, Australie). Les données codées ont été analysées pour en dégager des thèmes sémantiques afin de passer de la description pure des données à leur interprétation $^{15}$. Cette analyse a produit une description de l'emplacement des programmes mis en œuvre et des types de programme ainsi que des déterminants sociaux de la santé abordés. Le retour auprès des membres des organismes a été effectué en communiquant les résultats de l'analyse à au moins un organisme figurant dans l'analyse dans chaque province afin que leurs membres en relèvent les lacunes à l'échelle nationale ou dans leur région.

\section{Résultats}

L'analyse finale a porté sur 220 programmes (tableau 1). Les chiffres varient en fonction de la disponibilité de l'information et certains programmes ciblaient plusieurs populations. Un tiers des programmes $(34,5 \%)$ étaient non spécifiques et étaient offerts à toutes les personnes LGBTQ2 + . Dans les lieux regroupant de plus petits effectifs de population, c'était presque exclusivement le cas. La plupart des programmes $(65,5 \%)$ ciblaient certains groupes de personnes LGBTQ2 + spécifiquement, près de la moitié des programmes ciblant les jeunes. Même si la définition de " jeune " variait d'un organisme à l'autre, il s'agissait le plus souvent des 29 ans et moins. L'analyse a fourni peu de programmes pour les adultes de 55 ans et plus (données disponibles auprès des auteurs sur demande).

Le deuxième groupe le plus ciblé était celui des personnes ayant vécu une expérience trans. Environ $15 \%$ des programmes ciblés étaient axés sur les personnes transgenres et de diversité de genre, et certains étaient offerts par des organisations desservant uniquement cette

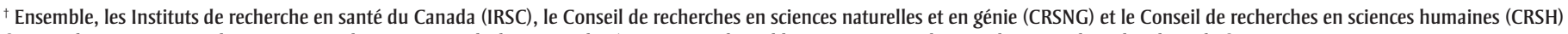
forment les organismes subventionnaires des trois Conseils, le principal mécanisme par lequel le gouvernement du Canada appuie la recherche et la formation. 
population. Ils étaient presque toujours axés sur l'offre de groupes de soutien, de soins de santé primaires ou de soutien à la navigation dans les systèmes de soins de santé, en particulier pour les soins d'affirmation de genre (aiguillage, accès aux hormones, chirurgies).

Environ $20 \%$ des programmes ont été conçus expressément pour les hommes gais, bisexuels et les autres hommes ayant des rapports sexuels avec des hommes (voir tableau 1) : il s'agit principalement d'organismes de services liés au VIH/sida. Les programmes ciblant les hommes étaient le plus souvent axés sur la santé sexuelle, certains portant néanmoins sur la santé sociale, la santé physique, la santé mentale et le bien-être général.

\section{Analyse}

Notre analyse a révélé des iniquités dans l'offre des programmes ${ }^{16}$. L'accent mis sur les programmes ciblant des groupes d'âge peut limiter la gamme de programmes offerts ${ }^{16}$. Cela peut avoir des répercussions sur la planification des systèmes de santé et les efforts de promotion de la santé parmi les membres de l'« intermédiaire absent $»^{16}$.

Moins de dix programmes ciblaient les Autochtones et les personnes bispirituelles ou les personnes LGBTQ2 + appartenant à une minorité raciale ou ethnique ${ }^{17-19}$. Il s'agissait souvent de groupes de soutien s'adressant à des personnes ayant une origine ethnique ou culturelle commune. Il y avait aussi peu de programmes (moins de 10) conçus spécifiquement pour les immigrants récents ou les réfugiés, et ceux qui existaient étaient localisés dans les grands centres urbains ${ }^{20}$. En outre, même si la recherche a été effectuée seulement en français et en anglais, seulement 7 programmes étant offerts dans une autre langue que les langues officielles ont été identifiés, ce qui pourrait constituer un obstacle important pour les locuteurs d'autres langues.

Un seul programme ciblait les personnes LGBTQ2 + vivant avec des déficiences neurocognitives.

Une minorité des programmes prenaient en compte les déterminants institutionnels et structurels importants de la santé en amont, comme l'emploi, l'éducation ou le logement ${ }^{17}$, secteurs où les personnes
TABLEAU 1

Résumé des résultats de l'analyse contextuelle des programmes ciblant les minorités de sexe et de genre, Canada, 2019

\begin{tabular}{|c|c|c|}
\hline Catégorie & n & $\%$ \\
\hline \multicolumn{3}{|l|}{ Localisation $(n=220)$} \\
\hline Ensemble du Canada & 16 & 7,3 \\
\hline Alberta & 29 & 13,2 \\
\hline Colombie-Britannique & 27 & 12,3 \\
\hline Manitoba & 15 & 6,8 \\
\hline Nouveau-Brunswick & 3 & 1,4 \\
\hline Terre-Neuve-et-Labrador & 3 & 1,4 \\
\hline Territoires du Nord-Ouest & 2 & 0,9 \\
\hline Nouvelle-Écosse & 9 & 4,1 \\
\hline Nunavut & 0 & 0 \\
\hline Ontario & 65 & 29,5 \\
\hline Île-du-Prince-Édouard & 2 & 0,9 \\
\hline Québec & 45 & 20,5 \\
\hline Saskatchewan & 3 & 1,4 \\
\hline Yukon & 1 & 0,5 \\
\hline \multicolumn{3}{|l|}{ Déterminants sociaux abordés $(n=220)$} \\
\hline Soutien social & 102 & 46,4 \\
\hline Exclusion sociale $^{\mathrm{a}}$ & 47 & 21,4 \\
\hline Accès aux services de santé & 51 & 23,2 \\
\hline Capacitisme & 1 & 0,5 \\
\hline Racisme, xénophobie et discrimination contre les immigrants & 11 & 5,0 \\
\hline Éducation & 2 & 0,9 \\
\hline Emploi & 2 & 0,9 \\
\hline Logement & 4 & 1,8 \\
\hline \multicolumn{3}{|l|}{ Taille de la collectivité $(n=220)$} \\
\hline Montréal, Toronto, Vancouver & 67 & 30,5 \\
\hline Grandes villes (population $>100000$ habitants) & 100 & 45,5 \\
\hline Petites villes (10 000 à 100000 habitants) & 19 & 8,6 \\
\hline Milieu rural (population $<10000$ ) & 2 & 0,9 \\
\hline Échelle provinciale & 16 & 7,3 \\
\hline Échelle nationale & 16 & 7,3 \\
\hline \multicolumn{3}{|l|}{ Langue $(n=213)$} \\
\hline Anglais & 155 & 72,8 \\
\hline Français & 26 & 12,2 \\
\hline Français et anglais & 32 & 15,0 \\
\hline Autre & 7 & 3,3 \\
\hline \multicolumn{3}{|l|}{ Niveau d'intervention $(\mathrm{n}=220)$} \\
\hline Promotion de la santéb & 41 & 18,6 \\
\hline Individuel et relations interpersonnelles & 128 & 58,2 \\
\hline Institutionnel & 47 & 21,4 \\
\hline Structurel & 4 & 1,8 \\
\hline
\end{tabular}

Suite à la page suivante 
TABLEAU 1 (suite)

Résumé des résultats de l'analyse contextuelle des programmes ciblant es minorités de sexe et de genre, Canada, 2019

\begin{tabular}{|c|c|c|}
\hline Catégorie & n & $\%$ \\
\hline \multicolumn{3}{|c|}{ Promotion de la santé et autres interventions à l'échelle individuelle selon le type de population ( $n=302)$} \\
\hline Bisexuel & 1 & 0,3 \\
\hline Invalidité & 1 & 0,3 \\
\hline $\begin{array}{l}\text { Homosexuels, bisexuels et autres hommes ayant des rapports } \\
\text { sexuels avec des hommes }\end{array}$ & 53 & 17,5 \\
\hline Personnes trans et de diversité de genre & 46 & 15,2 \\
\hline $\begin{array}{l}\text { Lesbiennes, bisexuelles et autres femmes ayant des rapports } \\
\text { sexuels avec des femmes }\end{array}$ & 10 & 3,3 \\
\hline LGBTQ & 46 & 15,2 \\
\hline Migrants et nouveaux arrivants & 7 & 2,3 \\
\hline Adultes plus âgés ${ }^{c}$ & 10 & 3,3 \\
\hline Parents, partenaires et autres soutiens & 30 & 9,9 \\
\hline Personnes racialisées & 12 & 4,0 \\
\hline Personnes bispirituelles & 6 & 2,0 \\
\hline Jeunes & 80 & 26,5 \\
\hline \multicolumn{3}{|l|}{ Spécificité de l'intervention ( $\mathrm{n}=220$ ) } \\
\hline Non spécifique & 76 & 34,5 \\
\hline Ciblée & 144 & 65,5 \\
\hline
\end{tabular}

Abréviation : LGBTQ2+, personne lesbienne, gaie, bisexuelle, transgenre, queer ou bispirituelle autochtone.

Remarque : Toutes les données n'étaient pas disponibles pour chaque programme et les chiffres entre les catégories ne sont donc pas comparables.

a Programmes qui favorisent l'inclusion des personnes LGBTQ2+ dans des espaces, des structures et des organismes non LGBTQ2+.

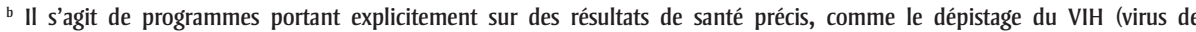
l'immunodéficience humaine) et des infections transmises sexuellement, la distribution de produits réducteurs des méfaits, les services de counseling et les services de lutte contre les dépendances.

c Seuls les programmes qui mentionnaient expressément l'âge des participants ont été comptabilisés dans ces catégories, c.-à-d. ceux pour les jeunes (moins de 30 ans) et ceux pour les adultes plus âgés (55 ans et plus).

LGBTQ2 + continuent à faire face à des obstacles importants en raison d'une stigmatisation et d'une discrimination persistantes. En effet, la majorité des interventions relevaient davantage de programmes en aval, ciblant l'accès aux soins de santé et visant l'échelle individuelle et interpersonnelle. Les programmes axés sur la santé ciblaient en grande partie les hommes et les personnes trans, les programmes liés à la santé destinés aux femmes cisgenres LGBTQ2 + étant particulièrement déficients ${ }^{21,22}$. D'autres recherches doivent porter sur la manière dont ces disparités affectent les résultats en santé.

Bien que des forces systémiques comme l'homophobie, la biphobie et la transphobie, le cishétérosexisme et d'autres systèmes d'oppression qui se cumulent créent des inégalités en matière de santé à tous les niveaux de l'écologie sociale, la plupart des programmes étant axés sur les niveaux individuels et interpersonnels d'intervention. Certains programmes peuvent être considérés comme relevant du niveau institutionnel, mais très peu d'entre eux fonctionnent réeelement à un niveau systémique ou structurel. Cette disparité empêche l'utilisation de stratégies et d'interventions aptes à réduire les obstacles liés à la stigmatisation des ressources sociales et matérielles auxquels font face les MSG $^{11,23,24}$. Pourtant, au Canada, les organismes LGBTQ2 + ainsi que d'autres organismes sociaux sont souvent financés par des institutions gouvernementales qui ciblent des interventions systémiques ou structurelles ${ }^{25}$. Les changements apportés au système pour faire progresser l'équité en santé par des interventions structurelles en amont sont sensibles aux efforts descendants (politiques, financement) comme ascendants (représentation), mais sont plus fructueux lorsqu'ils sont apportés simultanément et dans l'ensemble des secteurs $^{26,27}$.

\section{Conclusion}

Des efforts sont nécessaires pour mieux s'attaquer en amont aux déterminants de la santé affectant négativement les personnes LGBTQ2 + au Canada. Les efforts visant à élaborer de nouveaux programmes devraient viser les communautés LGBTQ2 + mal desservies par les services existants (en particulier les femmes, les Autochtones, les minorités raciales et ethniques, les personnes ayant récemment vécu l'immigration et les réfugiés). Le grand nombre de programmes de promotion du soutien social et de réduction de l'exclusion sociale donne à penser que ces programmes demeurent importants pour les utilisateurs finaux. Cela peut également être le reflet d'une préférence systémique en matière de financement pour les interventions en aval, par opposition à des interventions plus complexes et à long terme. Compte tenu de la rareté des interventions systémiques, les efforts futurs devraient être axés sur l'identification de pratiques prometteuses pour la conception, la prestation et l'évaluation d'interventions structurelles favorisant l'équité en santé et adaptées pour tenir compte des contextes particuliers des MSG.

\section{Remerciements}

Le financement de l'analyse a été fourni par la Division des déterminants sociaux de la santé de l'Agence de la santé publique du Canada. NJL reçoit une bourse d'études de la Fondation Michael Smith pour la recherche en santé $\left(\mathrm{n}^{\circ} 16863\right)$.

\section{Conflits d'intérêts}

Les auteurs déclarent n'avoir aucun conflit d'intérêts.

\section{Contributions des auteurs et avis}

BEJ et NJL ont conçu cette recherche et construit l'étude avec RH. BH a procédé à l'analyse générale, à l'analyse initiale des données et à la rédaction de la première version du manuscrit. RH a révisé l'analyse et a complété la première version du manuscrit. Tous les auteurs ont aidé à interpréter les données, ont révisé les différentes versions du manuscrit et en ont approuvé la version finale.

Le contenu et les points de vue exprimés dans cet article sont ceux des auteurs et ne reflètent pas nécessairement ceux du gouvernement du Canada.

\section{Références}

1. Lick DJ, Durso LE, Johnson KL. Minority stress and physical health among sexual minorities. Perspect Psychol Sci. 2013;8(5):521-548. https://doi.org/10 $.1177 / 1745691613497965$ 
2. Veale JF, Watson RJ, Peter T, Saewyc EM. Mental health disparities among Canadian transgender youth. J Adolesc Health. 2017;60(1):44-49. https://doi .org/10.1016/j.jadohealth.2016.09.014

3. Veale JF, Peter T, Travers R, Saewyc EM. Enacted stigma, mental health, and protective factors among transgender youth in Canada. Transgend Health. 2017;2(1):207-216. https://doi .org/10.1089/trgh.2017.0031

4. Russell ST, Ryan C, Toomey RB, Diaz RM, Sanchez J. Lesbian, gay, bisexual, and transgender adolescent school victimization: implications for young adult health and adjustment. J Sch Health. 2011;81(5):223-230. https://doi .org/10.1111/j.1746-1561.2011.00583.x

5. Dysart-Gale D. Social justice and social determinants of health: lesbian, gay, bisexual, transgendered, intersexed, and queer youth in Canada. J Child Adolesc Psychiatr Nurs. 2010; 23(1):23-28. https://doi.org/10.1111/j .1744-6171.2009.00213.x

6. Bogart LM, Revenson TA, Whitfield $\mathrm{KE}$, France CR. Introduction to the special section on Lesbian, Gay, Bisexual, and Transgender (LGBT) health disparities: where we are and where we're going. Ann Behav Med. 2014;47(1):1-4. https://doi.org/10.1007 /s12160-013-9574-7

7. Link BG, Phelan JC. Conceptualizing stigma. Annu Rev Sociol. 2001;27(1): 363-385. https://doi.org/10.1146 /annurev.soc.27.1.363

8. Casey LS, Reisner SL, Findling MG, et al. Discrimination in the United States: experiences of lesbian, gay, bisexual, transgender, and queer Americans. Health Serv Res. 2019;54(S2 Suppl 2):1454-1466. https://doi.org/10.1111 /1475-6773.13229

9. Hatzenbuehler ML. Structural stigma: research evidence and implications for psychological science. Am Psychol. 2016;71(8):742-751. https://doi.org /10.1037/amp0000068

10. Hatzenbuehler ML, Phelan JC, Link BG. Stigma as a fundamental cause of population health inequalities. Am J Public Health. 2013;103(5):813-821. https://doi.org/10.2105/AJPH.2012 .301069
11. L'équipe de Trans PULSE Canada. Rapport Trans PULSE Canada. Accès à la santé et aux soins de santé pour les personnes trans et non binaires au Canada : résultats nationaux, provinciaux et territoriaux [Dans Internet]. Trans PULSE Canada; 10 mars 2020 [consulté le 3 mai 2021]. En ligne à : https://transpulsecanada.ca/fr/research -type/rapports/

12. Réseau pancanadien de santé publique. Les principales inégalités en santé au Canada : un portrait national. Ottawa (Ont.) : Agence de la santé publique du Canada; 2018. En ligne à : https://epe .lac-bac.gc.ca/100/201/301/weekly_ acquisitions_list-ef/2019/19-06 /publications.gc.ca/collections/collection _2019/aspc-phac/HP35-109-2018-1-fra.pdf

13. Veenstra G. Race, gender, class, and sexual orientation: intersecting axes of inequality and self-rated health in Canada. Int J Equity Health. 2011; 10(1):3. https://doi.org/10.1186/1475 $-9276-10-3$

14. Chambre des communes. La santé des communautés LGBTQIA2 au Canada. Rapport du Comité permanent de la santé. Ottawa (Ont.) : Chambre des communes; 2019. https://www.ourcommons .ca/Content/Committee/421/HESA /Reports /RP10574595/hesarp28 /hesarp28-f.pdf

15. Braun V, Clarke V. Using thematic analysis in psychology. Qual Res Psychol. 2006;3(2):77-101. https:// doi.org/10.1191/1478088706qp063oa

16. Fredriksen-Goldsen KI. Promoting health equity among LGBT mid-life and older adults: revealing how LGBT mid-life and older adults can attain their full health potential. Generations. 2014; 38(4):86.

17. Mulé NJ, Ross LE, Deeprose B, et al. Promoting LGBT health and wellbeing through inclusive policy development. Int J Equity Health. 2009;8(1):18 https://doi.org/10.1186/1475-9276-8-18

18. Hunt S. Une introduction à la santé des personnes bispirituelles : questions historiques, contemporaines et émergentes. Prince George (C.-B.) : Centre de collaboration nationale de la santé autochtone; 2016. En ligne à : https:// www.ccnsa-nccah.ca/docs/emerging /RPT-HealthTwoSpirit-Hunt-FR.pdf
19. Battle J, Ashley C. Intersectionality, heteronormativity, and Black lesbian, gay, bisexual, and transgender (LGBT) families. Black Women Gender Families. 2008;2(1):1-24. www.jstor.org/stable /10.5406/blacwomegendfami.2.1.fm

20. Munro L, Travers R, John AS, et al. A bed of roses?: Exploring the experiences of LGBT newcomer youth who migrate to Toronto. Ethn Inequal Health Soc Care. 2013;6(4):137-150. https:// doi.org/10.1108/EIHSC-09-2013-0018

21. Steele LS, Daley A, Curling D, et al. LGBT identity, untreated depression, and unmet need for mental health services by sexual minority women and trans-identified people. J Womens Health (Larchmt). 2017;26(2):116-127. https://doi.org/10.1089/jwh.2015.5677

22. Operario D, Gamarel KE, Grin BM, et al. Sexual minority health disparities in adult men and women in the United States: National Health and Nutrition Examination Survey, 20012010. Am J Public Health. 2015;105(10): e27-34. https://doi.org/10.2105/AJPH .2015 .302762

23. Oldenburg CE, Perez-Brumer AG, Hatzenbuehler ML, Krakower D, Novak DS, Mimiaga MJ, Mayer KH. State-level structural sexual stigma and HIV prevention in a national online sample of HIV-uninfected men who have sex with men in the United States. AIDS. 2015;29(7):837.

24. Chaudoir SR, Wang K, Pachankis JE. What reduces sexual minority stress? A review of the intervention "toolkit". Journal of Social Issues. 2017 Sep; 73(3):586-617.

25. Clément D. State funding for human rights activism: channeling protest? Am Behav Sci. 2017;61(13):1703-1728. https://doi.org/10.1177/00027642177 44133

26. Farrer L, Marinetti C, Cavaco YK, Costongs C. Advocacy for health equity: a synthesis review. Milbank Q. 2015;93(2):392-437. https://doi.org /10.1111/1468-0009.12112 
27. Carey G, Crammond B, Keast R. Creating change in government to address the social determinants of health: how can efforts be improved? BMC Public Health. 2014;14(1):1087. https://doi.org/10.1186/1471-2458 $-14-1087$ 